A component based approach for classifying the seven universal facial expressions of emotion

by Kenny Hong, Stephan K. Chalup and Robert A. King

Copyright (C) 2013 IEEE.

This is an author-prepared version of the article, reprinted from Proceedings of 2013 IEEE Symposium on Computational Intelligence for Creativity and Affective Computing (CICAC) (Singapore 16-19 April, 2013) p. 1-8

\title{
http://dx.doi.org/10.1109/CICAC.2013.6595214
}

This material is posted here with permission of the IEEE. Such permission of the IEEE does not in any way imply IEEE endorsement of any of University of Newcastle's products or services. Internal or personal use of this material is permitted. However, permission to reprint/republish this material for advertising or promotional purposes or for creating new collective works for resale or redistribution must be obtained from the IEEE by writing to pubs-permissions@ieee.org. By choosing to view this document, you agree to all provisions of the copyright laws protecting it. 


\section{A Component Based Approach for Classifying the Seven Universal Facial Expressions of Emotion}

\author{
Kenny Hong \\ School of Electrical Engineering and \\ Computer Science \\ The University of Newcastle, Australia \\ Email: kenny.hong@uon.edu.au
}

\author{
Stephan K. Chalup \\ School of Electrical Engineering and \\ Computer Science \\ The University of Newcastle, Australia \\ Email: stephan.chalup@newcastle.edu.au
}

\author{
Robert A.R. King \\ School of Mathematical and \\ Physical Sciences \\ The University of Newcastle, Australia \\ Email: robert.king@ newcastle.edu.au
}

\begin{abstract}
In a previous publication we showed a component approach performing better than a holistic approach for classifying 6 discrete facial expressions of emotion (happy, sad, surprised, fearful, disgusted and angry) plus the neutral face. In this paper, we present the impact on the two approaches if a seventh facial expression is included. This seventh expression is known as the contemptuous expression (an expression that has rarely been explored in the machine learning literature). Together, the 7 facial expressions are known in the psychology literature as Ekman's Universal Facial Expressions of Emotion. To replicate our previous experiments, we use the same number of image preprocessing techniques (grayscale, Local Binary Patterns, Sobel and Canny edge detection, and manually inserted feature points which we refer to as components) that are organised into four approaches: holistic, holistic action, component and component action (where an action approach is produced by a difference between a neutral and an expressive face). The facial expressions are classified using a standard multiclass Support Vector Machine (SVM) and a pairwise adaptive multiclass SVM (pa-SVM) - which uses pairwise adaptive model parameters. The significance of this study is to provide a thorough understanding of the choices that impact a classifier's performance and the performance dynamics between expression pairs when the contemptuous expression is now considered. In particular, we extend the issues and justify the use of the contemptuous expression by noting its universal presence in human expression and its asymmetric nature, which is not shared with other expressions. Using our face model, which uses feature points around the eyes, brows and mouth, we obtained a best correct classification rate of $98.57 \%$ with the contemptuous expression included.
\end{abstract}

\section{INTRODUCTION}

Facial expression classification has gained popularity because of its potential for applications in human computer interaction (HCI) - where the ability to automate the capture of emotions, personality traits and cognitive intentions from facial expressions [15] would be a significant advancement [21]. In a previous publication [20] we showed a component approach performing better than a holistic approach for classifying 6 discrete facial expressions of emotion (happy, sad, surprised, fearful, disgusted and angry) plus the neutral face. In this paper, we present the influence on the two approaches if a seventh facial expression is included. This seventh expression is known as the contemptuous expression. It is the only facial expression that is expressed asymmetrically and has rarely been explored in the machine learning literature.
We begin with an overview of the facial expressions used most commonly in the machine learning literature. Next we present our reasons as to why the contemptuous expression is considered an important expression to be included in future studies, which motivates our study.

Happy, sad, surprised, fearful, angry and disgusted are the typical 6 discrete expressions that have been used in many facial expression studies [25, 31, 44]; only a few studies have included a contemptuous expression [2, 6, 30, 35]. Paul Ekman and Wallace V. Friesen have described how these 7 expressions are universally recognised [10, 16], and have appropriately named them the 'Universal Facial Expressions of Emotion'. In addition, they have devised a widely used system of metrics known as 'The Facial Action Coding System' (FACS) which captures facial actions that are applicable to facial expressions [11]. Returning to the choices of discrete expressions, the neutral face has been included by some researchers as an additional expression to classify [18, 24, 25, 27-29, 31, 43, 45] while others analyse the difference images that are produced by subtracting the neutral face from an expressive face $[4,5$, 33, 40, 42, 47].

Ekman describes the contemptuous expression as a tightening and slight raising of the lip corner, primarily on one side of the face [12]. This visual asymmetry separates it from the rest of the facial expressions. A primary question is 'What makes the contemptuous expression a minority?'. Critics such as Russell have mentioned that the contemptuous expression is the least well established of the universal expressions [36]. One controversy is the expression being mistaken to be disgusted ([36]) or a variant of disgusted ([39]). Russell questions the validity of Ekmans force choice methods, and Ekman later responds to Russell's work as being a mistaken critique [13].

There are a number of studies that support the universality of the contemptuous expression and its separation from the disgusted expression. For instance, Ekman's initial study in 1986 reported an average recognition of $75 \%$ across 10 cultures for the contemptuous expression [16]. Ekman's replicated study with Heider in 1988 [12] showed strong agreement, as did supporting studies made by Matsumato in 1992 [32].

From what we have described, the evidence supporting the contemptuous expression and its visual asymmetry when com- 
pared to the typical 6 discrete expressions merits our curiosity in regards to the impact it could have on a classifier's performance. Our aim is to replicate the experiments of our previous publication [20] with the inclusion of the contemptuous expression to explore its influence on a classifier's performance. The goal of our previous publication was to understand the impact of a classifier's performance using a number of image preprocessing techniques (grayscale, Local Binary Patterns, Sobel and Canny edge detection, and manually inserted feature points which we refer to as components) that are organised into four approaches: holistic, holistic action, component and component action (where an action approach is produced by a difference between a neutral and an expressive face). The manually inserted feature points became our proposed face model. Further image preprocessing techniques and justifications for using datasets certified by facial expression experts were also presented in the literature review of our previous publication. To replicate our previous experiments, we use the same number of image preprocessing techniques and our face model. The facial expressions are classified using a Gaussian kernel with a standard multiclass Support Vector Machine (SVM) and a pairwise adaptive multiclass SVM (pa-SVM), which uses pairwise adaptive model parameters [19].

A system overview is presented in the next section, followed by a description of the dataset and image preprocessing used in section III. Section IV presents the classification method followed by the experimental results and discussion in Section V. We provide our concluding remarks in Section VI.

\section{SYSTEM OVERVIEW}

Our system consists of several steps as illustrated in Figure 1. Our first step is to manually insert feature points for each face in the dataset so that the faces can be aligned. After image preprocessing we have four datasets: grayscale, Local Binary Patterns, edges using Canny and Sobel operator, and feature points. The differences between the expressive and neutral face are produced for each dataset. The resultant datasets are then organised into four approaches: holistic, holistic action, component and component action. Details of each module and further steps are explained in subsequent sections.

\section{DATASET AND IMAGE PREPROCESSING}

In this section we describe our face dataset, how it was aligned using our face model, and the image preprocessing techniques used.

Our face datasets are generated using the JACFEE and JACNEUF dataset, which is certified by the Paul Ekman Group LLC [34]. We specifically chose this dataset because it has discrete expression classes (the 7 universal facial expressions of emotion as well as neutral faces). The dataset consists of 280 coloured face images, including 140 neutral faces and 20 images of 7 different facial expressions. 60 feature points were manually inserted for each face image along the brows, eyes and mouth. These feature points became our face model and we use 2 feature points of the inner eye corners (a.k.a lacrimal caruncle) for aligning our face dataset. Once aligned

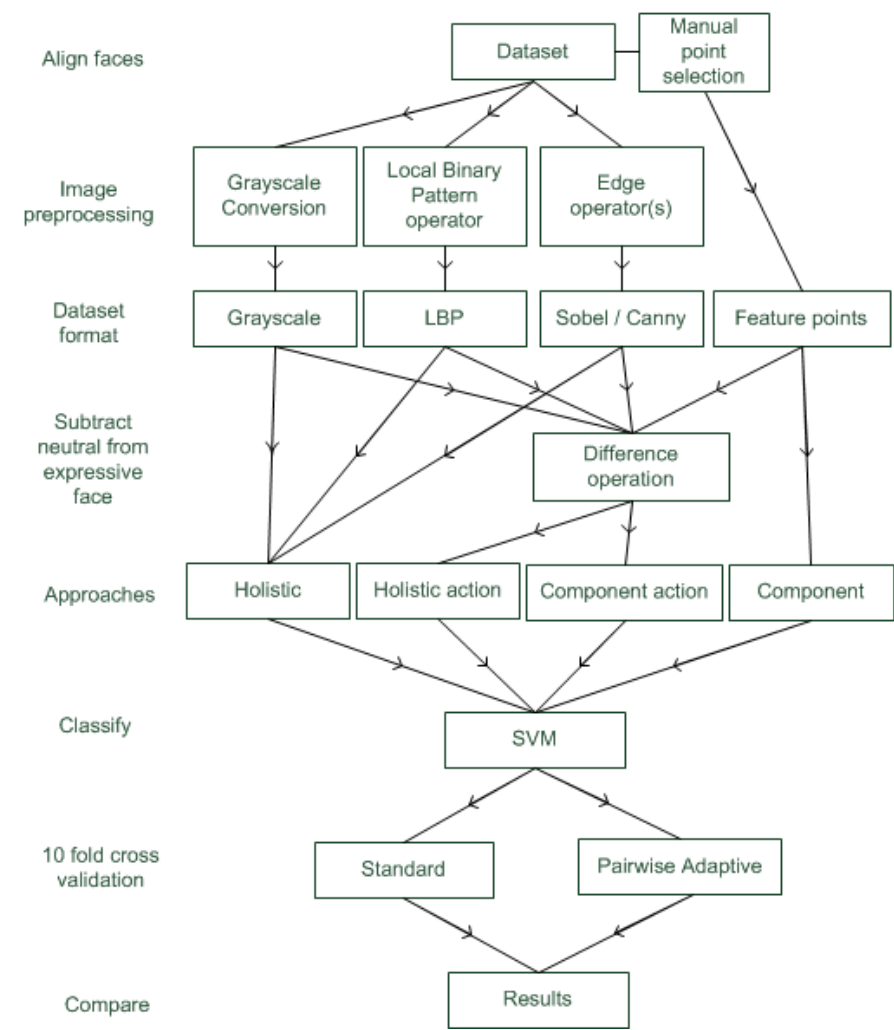

Fig. 1: System overview showing the flow of the stages and states.

the images are cropped and resized to $100 \times 100$ pixels for image preprocessing - results of a face crop and a head crop are obtained and discussed in Section V.

\section{A. Proposed face model}

Returning to our face model, we present a revised description of our previous publication [20]. An illustration of our face model is provided in Figure 2. In this illustration the feature points are coloured red, blue and green representing respectively a stable, active or passive state during an expression. The reasons for our chosen feature points are as follows:

1) Stable: We chose the inner eye corners, otherwise known as the lacrimal caruncle because they are invariant across facial expressions, and as they are stable points they are ideal for aligning our face dataset.

2) Active: We chose the brow end points, mid-upper and mid-lower eyelids, lip end points, and mid-upper and mid-lower lips because we consider them to be the minimal set of points required to describe and distinguish between expressions - i.e. the differences of these points between expressions are the largest.

3) Passive: We consider passive points to be the points that are positioned between active points. Similar to active points, they do vary between expressions, however they are not essential in separating one expression from the next. They are there to enhance the shape of the brows, eyes and mouth movements.

Affine transformations: The individual face images are aligned via translation, rotation and scaling using the inner 


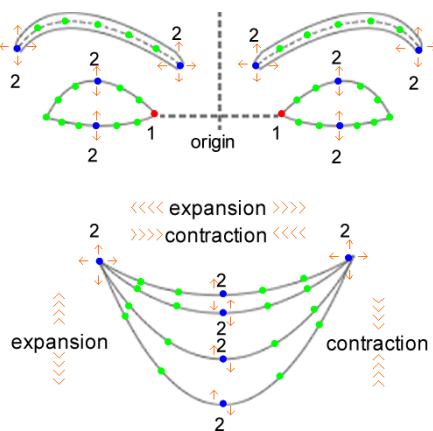

Fig. 2: Face model showing the feature points $[1=$ stable, $2=$ active, and the remainders are passive], facial actions and directions of horizontal and vertical expansion and contraction.

eye corners such that they lie on the horizontal $x$-axis and the midpoint between the inner eye corners sits on the origin of the transformed plane. The transformation matrix is as follows:

normalise $(x, y)=\operatorname{scale}(x, y) \times \operatorname{rotate}(x, y) \times \operatorname{translate}(x, y)$

$$
\operatorname{translate}(x, y)=\left[\begin{array}{c}
-\frac{\mathrm{x}_{L}+\mathrm{x}_{R}}{2} \\
-\frac{\mathrm{y}_{L}+\mathrm{y}_{R}}{2}
\end{array}\right]\left[\begin{array}{l}
x \\
y
\end{array}\right]
$$

where $\mathrm{x}_{L}, \mathrm{x}_{R}, \mathrm{y}_{L}, \mathrm{y}_{R}$ are the left and right inner eye corners.

$$
\operatorname{rotate}(x, y)=\left[\begin{array}{cc}
\cos (-\theta) & -\sin (-\theta) \\
\sin (-\theta) & \cos (-\theta)
\end{array}\right]\left[\begin{array}{l}
x \\
y
\end{array}\right]
$$

where $\theta$ is the angle between the interval joining the inner eye corners and the horizontal $x$-axis.

$$
\operatorname{scale}(x, y)=\frac{1}{2 \mathrm{x}_{R}}\left[\begin{array}{l}
x \\
y
\end{array}\right]
$$

where $\mathrm{x}_{R}$ is right eye corner.

\section{B. Holistic approach}

Four image preprocessing techniques were used: grayscale, Local Binary Patterns (LBP), edges and feature points. As feature points have been described previously, a description of the first three is as follow:

1) Grayscale: The grayscale uses an ITU-R 601-2 luma transform on RGB (red green blue) colours [1]:

$$
\text { grayscale }=\text { red } * 0.299+\text { green } * 0.587+\text { blue } * 0.114
$$

2) Local Binary Patterns: The Local Binary Pattern (LBP) uses a $3 \times 3$ neighborhood LBP operator [40]; and is a nonparametric transform.

3) Edges using Canny and Sobel: The Canny and Sobel edge operators were used for our edge images. Both operators have several parameters that impact the final edge image. Selection of the Sobel and Canny parameters was by visual evaluation for ideal face edges. The parameters and the ranges are as follows-lower threshold: 85 [0-255] and upper threshold: 170 [0-255] for Sobel and Canny, and for Sobel only a blur of 0 [0-50] and a gain of 5 [1-10].
The grayscale, Local Binary Patterns and edges represent our holistic approach. To obtain the holistic action approach we subtract the neutral faces from the expressive faces. Examples of our holistic and holistic action approach are shown in Figure 3.

1. contempt.
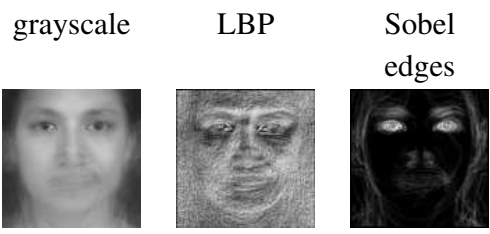

Canny
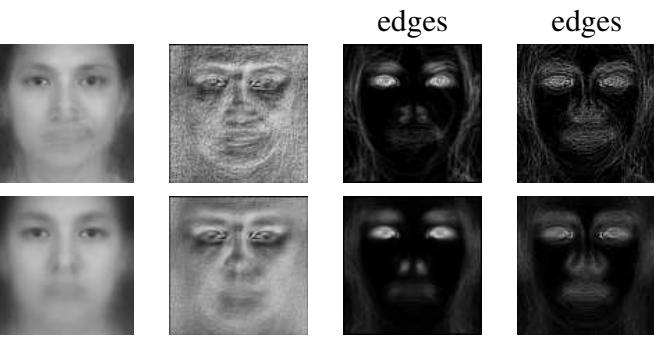

2. neutral
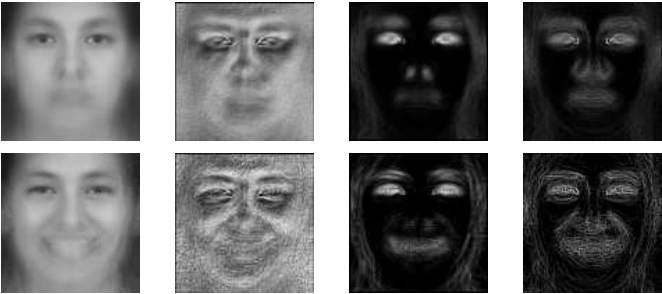

3. happy
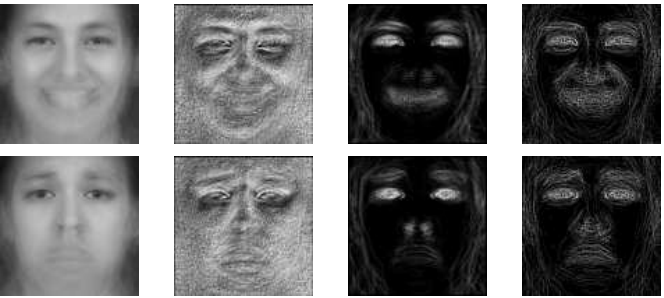

4. sad
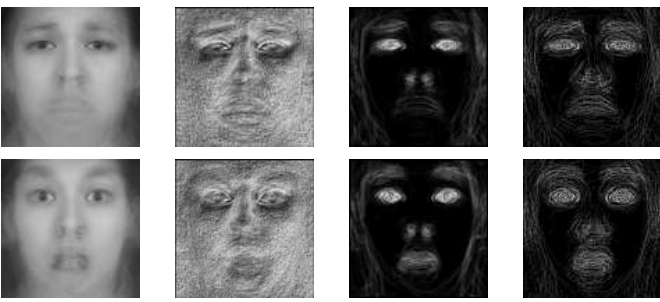

5. surprised
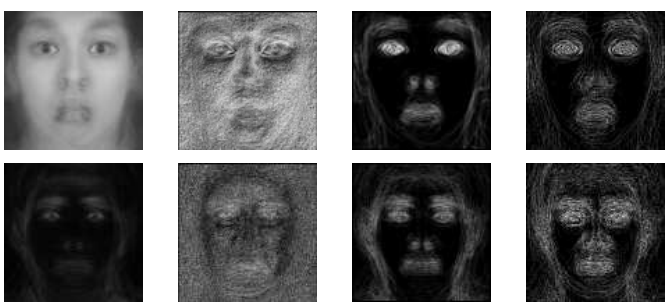

6. fearful
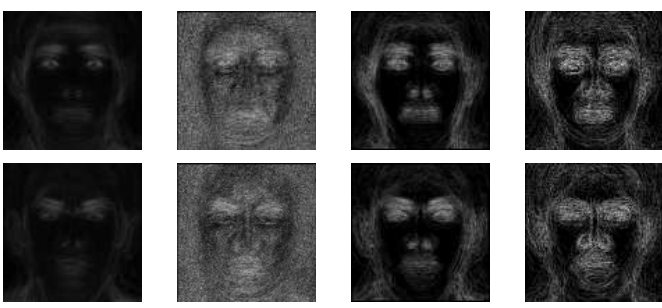

7. angry
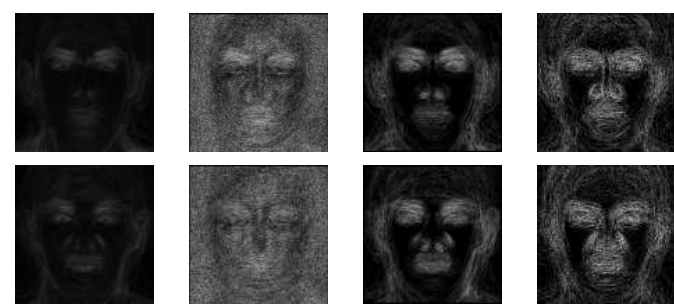

8. disgusted

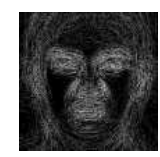

Fig. 3: Examples of our holistic and holistic action datasets. The average of each expression is shown from rows 1 to 8 in their respective preprocessed images. The use of the inner eye corners for alignment of faces is evident by the clarity around that area. Rows 15 show a face crop; while rows 6-8 show a head crop and our holistic action approach where the difference images between an expressive and a neutral face are averaged. The contemptuous expression in row 1 shows the visual asymmetry of this facial expression. Our face datasets are generated from the JACFEE and JACNEUF dataset [34].

\section{Component approach}

Our previously described face model and its feature points form our component approach. To obtain the component action approach we use the differences between the feature points of an expressive and a neutral face, which we refer to as facial actions.

The facial actions assigned to each feature point are shown in Table I and illustrated in Figure 2. In the table (I), we 
TABLE I: Facial actions assigned to feature points for the face model of Figure 2. When we refer to the upper or lower lip we mean both the inner edge and outer edge of the lip.

\begin{tabular}{|l|l|}
\hline Feature points & Actions \\
\hline brow endpoints & left, right, up, down \\
midpoint of upper \& lower eyelids & up, down \\
mouth corner points & up, down, left, right \\
midpoint of inner edge upper lip & up, down \\
midpoint of inner edge lower lip & up, down \\
inner edge upper lip & horizontal contr./expansion \\
inner edge lower lip & horizontal contr./expansion \\
midpoints of upper lip & vertical contr./expansion \\
midpoints of bottom lip & vertical contr./expansion \\
\hline
\end{tabular}

describe four primary facial actions: left, right, up and down; and secondary facial actions: horizontal and vertical contraction and expansion. The horizontal contraction and expansion uses the difference of the sum of the distances between the active and passive feature points along the inner edge of the lips; while the vertical contraction and expansion uses the difference of the distance between the midpoints of the inner and outer edges of the lips. The horizontal and vertical facial actions were introduced to describe the mouth more clearly as it has a higher degree of movement than the brows and the eyes. The equation is as follows:

$$
\operatorname{diff}=\sum_{i=0}^{n-1}\left(E_{i+1}-E_{i}\right)^{2}-\sum_{i=0}^{n-1}\left(N_{i+1}-N_{i}\right)^{2}
$$

where $E_{i, i=0, \ldots, n-1}$ represents the feature points of an expressive face, $N_{i, i=0, \ldots, n-1}$ represent the feature points of a neutral face, and diff indicates an expansion if diff $>0$ and a contraction if $\operatorname{diff}<0$.

\section{ClasSIFICATION OF FACIAL EXPRESSIONS}

In our previous publication [20] we use Support Vector Machines for multiclass classification as they are the most effective and commonly used tools for facial expression classification [5, 9, 23, 30, 40, 48]. We also assess our proposed modified multiclass SVM known as 'Pairwise Adaptive SVM' (pa-SVM, presented in [19]) on the 7 universal facial expressions of emotion. Likewise, we use the Gaussian kernel with the one-against-one multiclass framework because of its suitability for application to a large multiclass dataset [8].

\section{A. The SVM and kernel parameters to optimise}

The SVM with a Gaussian kernel has two parameters to optimise: $C>0$ and $\gamma>0$ (Gamma). $C>0$ adjusts the penalty of the error term that maximises margin separation by minimising the primal form objective function [41]:

$$
\min _{\mathbf{w}, \xi}\left(\frac{1}{2} \mathbf{w}^{T} \mathbf{w}+C \sum_{i=1}^{m} \xi_{i}\right)
$$

subject to the constraints:

$$
\begin{aligned}
y_{i}\left(\mathbf{w}^{T} \Phi\left(\mathbf{x}_{i}\right)+b\right) & \geq 1-\xi_{i} \\
\xi_{i} & \geq 0
\end{aligned}
$$

where $\mathbf{w}$ is the normal vector, $b$ is the bias, $\xi_{i} \geq 0$ are the slack variables, $\mathbf{x}_{i}$ are the samples and $\mathbf{y}_{i}$ are the labels $( \pm 1)$; while $\gamma>0$ adjusts the Gaussian kernel function:

$$
K\left(\mathbf{x}_{i}, \mathbf{x}_{j}\right)=e^{-\gamma\left\|\mathbf{x}_{i}-\mathbf{x}_{j}\right\|^{2}} \quad \gamma>0
$$

\section{B. pa-SVM Implementation}

The current implementation of $p a-S V M$ for this paper is the same as our previous publication [20] where we used the LIBSVM library for the one-against-one multiclass strategy and grid search as they are already present [7]. The grid search used in LIBSVM is a coarse to fine search that grows the sequences of $C$ and $\gamma$ exponentially. What is different to the standard multiclass SVM is that individual $(C, \gamma)$ pairs are assigned to each binary classifier and their best values are determined using the LIBSVM grid search.

\section{Feature vectors}

Four feature vectors were defined for each of the approaches. The holistic and holistic action have a feature vector of dimension $100 \times 100$ using the image intensities; the component approach has a feature vector of dimension 120 using the $x y$ coordinates of the 60 feature points; and the component action approach has a feature vector of dimension 44 covering the facial actions described in Table I.

With the exception of the component approach all other feature vectors were normalised between -1 and +1 .

\section{RESUlts AND Discussion}

An overview of the experimental results is shown in Table II. Looking at the summary table, we can see where perfor-

\begin{tabular}{|c|c|c|}
\hline Approaches & Include contempt & Exclude contempt \\
\hline \multirow{2}{*}{ holistic } & $\begin{array}{c}\text { Include neutral: } \\
\text { grayscale }>\text { Sobel }> \\
\text { Canny } \geq \text { LBP }\end{array}$ & $\begin{array}{c}\text { Include neutral: } \\
\text { grayscale }>\text { Sobel }> \\
\text { Canny } \geq \text { LBP }\end{array}$ \\
\hline & $\begin{array}{c}\text { Exclude neutral: } \\
\text { Sobel }>\text { LBP }> \\
\text { Canny }>\text { grayscale }\end{array}$ & $\begin{array}{l}\text { Exclude neutral: } \\
\text { LBP }>\text { Canny }> \\
\text { Sobel }>\text { grayscale }\end{array}$ \\
\hline holistic action & $\begin{array}{l}\text { grayscale }>\text { LBP }> \\
\text { Sobel }>\text { Canny }\end{array}$ & $\begin{array}{l}\text { grayscale }>\text { LBP }> \\
\text { Canny }>\text { Sobel }\end{array}$ \\
\hline component & \multicolumn{2}{|c|}{ exclude neutral $>$ include neutral } \\
\hline component action & \multicolumn{2}{|c|}{$\begin{array}{l}\text { include horizontal and vertical } \\
\text { expansion and contraction returns higher result }\end{array}$} \\
\hline Summary & \multicolumn{2}{|c|}{$\begin{array}{ll}\text { cropping: } & \text { face crop }>\text { head crop } \\
\text { contempt: } & \text { exclude }>\text { include } \\
\text { neutral: } & \text { exclude }>\text { include } \\
\text { approaches: } & \begin{array}{c}\text { component action }>\text { component }> \\
\quad \text { holistic }>\text { holistic action }\end{array}\end{array}$} \\
\hline
\end{tabular}
mances vary among different image preprocessing of different approaches with and without the contemptuous expression. We discuss the results of these tables further in subsequent subsections.

TABLE II: Overview of the experimental results. We can see where performances vary among different image preprocessing of different approaches with and without the contemptuous expression. A summary of the overall performances is shown at the bottom of the table. 


\section{A. Performances under cropping and different image prepro- cessing}

The first notable impact is that cropping closer to the face returns better results for both the holistic and component approaches. Returning to the summary table (II) the top performer for the holistic approaches was grayscale except when the neutral face was excluded. However, grayscale, Sobel, Canny and LBP cover only the holistic approaches.

The feature points of the component approach returned the best rates. One reason is the minimal noise when compared to other preprocessing techniques, and no requirement for tuning of parameters. However, the feature points need to be manually inserted. On the other hand, tuning the edge operator's parameters is required in the holistic approach and it affects the visual clarity of the facial features of the final edge image. Our parameter selection was by visual clarity of these features as there are currently no literature on determining the optimal parameters for edge filtering on face images.

\section{B. Component is the better approach}

The component action approach returned the best result with an accuracy of $98.57 \%$. This was followed by the component approach with $80.83 \%$ best accuracy and the holistic approach with a best accuracy of $71.15 \%$. Least accurate was the holistic action approach with a best of $62.50 \%$. The reported percentages are from the proposed pairwise adaptive multiclass SVM, which performed favourably in many of the experiments conducted.

Extending our previous publication [20], there is still an on-going debate between the merits of the holistic and component approaches [46]. Our results indicate that a component approach improves classification of discrete facial expressions over a holistic approach. Could it be the influence of holistic approaches that are dominant in the area of facial recognition? When face recognition research was first introduced, the primary methods relied mostly on feature points and distances between them [38]. This resulted in poor recognition, and researchers began looking into holistic methods that dealt with intensity values such as grayscale [3]. Then facial expression studies emerged at the peak popularity of holistic methods [22] and soon a large set of literature on holistic approaches for facial expression analysis followed [17, 46].

\section{Excluding the contemptuous expression and neutral does impact performance}

As mentioned in our introduction, the contemptuous expression and neutral have been excluded in most of the classification studies, with the former being covered substantially less. Our analysis of the results from the holistic and component approaches show that when the contemptuous expression and neutral are excluded, the overall classification performance improves. We examine these two expressions more closely.

The neutral face is difficult to classify. One reason for this is its subtle resemblance to other facial expressions, and this can be linked to the overgeneralisation of an emotion recognition system [37]. To analyse this impact we use a two class SVM with a Gaussian kernel to compare the neutral face with the rest of the expressions. From our experiment, a high rate of $74.29 \%$ for the holistic and $88.21 \%$ for the component approach suggests that SVMs are capable of distinguishing between a neutral and an expressive face. These results are reflected in the Receiver Operator Characteristic Curves (ROC) of Figure 4. This is a slightly lower return rate than our previous publication without the contemptuous expression [20], where a high rate of $76.15 \%$ for the holistic and $88.85 \%$ for the component approach was returned.

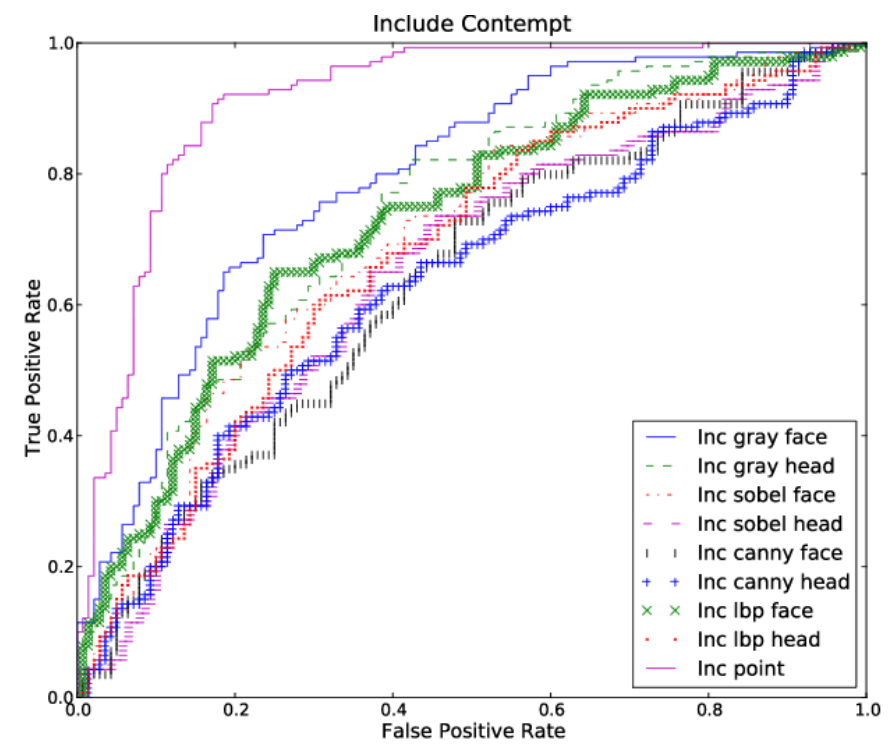

Fig. 4: ROC curves. The component approach (i.e. feature points) is the best performer for distinguishing between a neutral and an expressive face. This is shown by the 'Inc point' where 'Inc' is including the contemptuous expression.

\section{The contemptuous expression is conceived from a literate culture}

We have referenced several studies in our introduction that affirm the universality of the contemptuous expression. In [14] Ekman cited that his universality of the contemptuous expression was conceived by evidence from literate cultures, as locating an isolated preliterate culture was not possible. Ekman also mentioned that 'fearful and surprised' was only distinguishable in literate cultures. In this study we assume an application for a literate culture, and base on this assumption a fearful expression can be distinguished from a surprised expression and the contemptuous expression is universally recognised.

\section{E. Is there an optimal parameter pair?}

Similar to our previous publication [20], a question that we consider to be relevant in this study is whether there is an optimal parameter pair in the parameter space for classifying expressions. To address this question, we use Figure 5 and 6, which plot (respectively) the $C, \gamma$ and correct classification rates of all the approaches for the standard and proposed 
multiclass SVMs. Each approach in the figures is represented by colour coded circles and the largest circles represent the most frequent pair.

Looking at Figure 5, the standard multiclass SVM has only 2 repeated $C$ and $\gamma$ values of $\log 2(3,-15)$ and $\log 2(9$,15) with classification rates of $38.57 \%$ and $61.43 \%$ using a holistic action approach. This small amount is not considered an optimal pair. However, the pairwise adaptive multiclass SVM has 54 repeated $C$ and $\gamma$ values of $\log 2(-5,-7)$ with classification rates of $100 \%$ (component approach) and $87.5 \%$ (holistic approach). In both figures, the component action approach is the best performer as shown at the top layer of the plots.

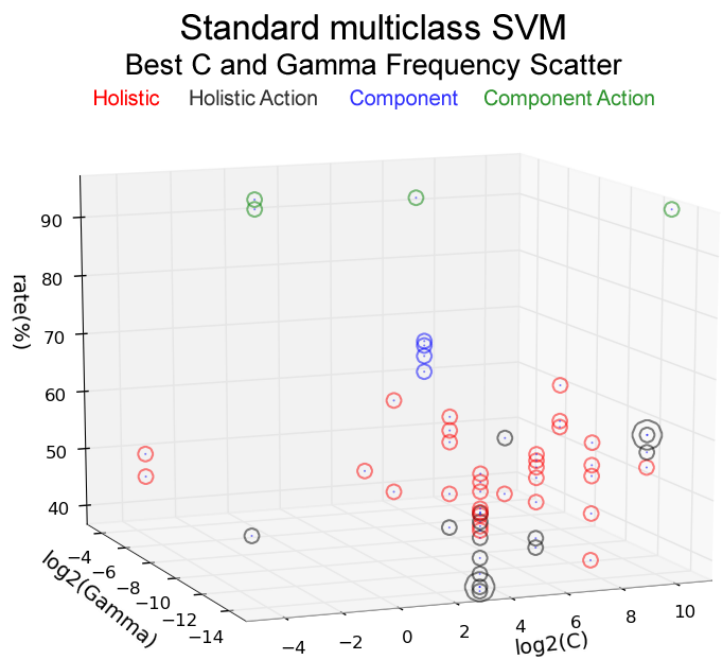

Fig. 5: This graph displays the correct classification rate of $(C, \gamma)$ pairs for all experiments of the standard multiclass SVM where each approach is shown by colour coded circles.

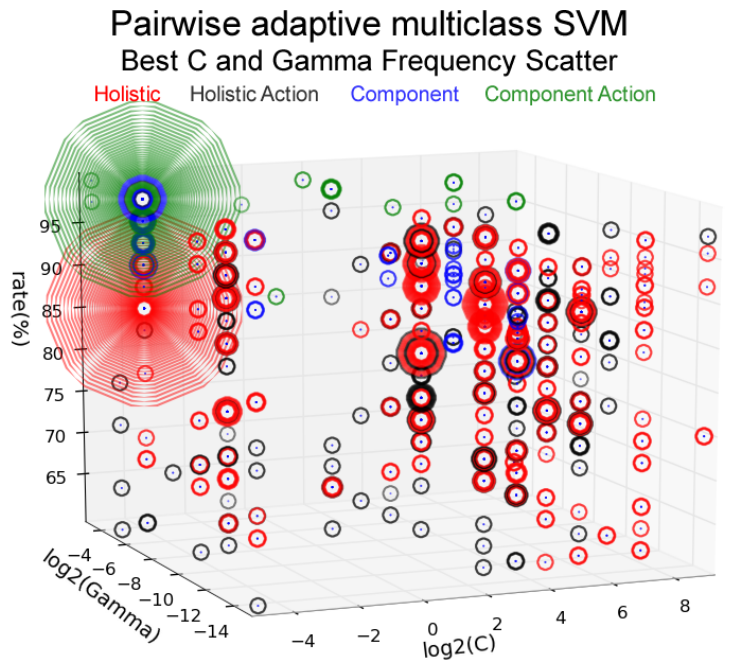

Fig. 6: In the pairwise adaptive multiclass SVM approach a $(C, \gamma)$ pairing can occur several times in one experiment. The frequency of $(C, \gamma)$ pairs is indicated by the number of increasing circles. The most frequently occuring pair is $\log 2(-5,-7)$ with classification rates of $100 \%$ (component approach) and $87.5 \%$ (holistic approach).

\section{F. Performance dynamics of expression pairs suggest a non equidistant expression space}

Revising our previous publication [20], the topic of expression space based on discrete facial expressions was investigated $[29,43]$. In Table III, we present the performance dynamics of expression pairs where each rate is an average of all the experiments using the $p a-S V M$ method. By examining this table (III), the results of the paired expressions indicate that some pairs of expressions are easier to distinguish than others. This suggests an expression space where expressions are not equidistant. For instance, the fearful and surprised pair in Table III returned the lowest average rate while the happy and surprised pair gave the best average rate. The poor performance of the fearful and surprised pair corresponds to the difficulty in distinguishing the two expressions that is apparent in preliterate cultures [14] - suggesting that the expression space of fearful and surprised has a larger overlapped region than that of happy and surprised.

Further, these results agree with studies such as [5] which showed that human participants performed poorly at separating anger and disgust while happy and surprised were easily distinguished. In the same study, they excluded sad and fear as they decided that these expressions were not as distinctive as the other basic expressions. Our fear-sad expression pair in Table III agrees with their decision, as it is ranked third last. Examining the table specifically on the contemptuous expression (because of its visual asymmetry), the table shows that the classifier can easily distinguish it from surprised, but only poorly distinguish it from angry.

The above mentioned studies with human participants cover only a small subset of our results shown in Table III. An extensive literature review shows a lack of human participant research for the remaining expression pairs in Table III.

\begin{tabular}{|l|l|l|l|l|l|l|l|}
\hline Pair & Rate & Pair & Rate & Pair & Rate & Pair & Rate \\
\hline hap,sur & 96.88 & sur,dis & 90.98 & sad,neu & 87.88 & con,sad & 81.25 \\
hap,fea & 93.75 & fea,neu & 90.69 & ang,fea & 87.59 & hap,dis & 80.45 \\
sur,ang & 93.04 & ang,neu & 90.25 & hap,ang & 87.05 & hap,con & 78.93 \\
sur,con & 92.32 & hap,sad & 89.82 & ang,sad & 85.09 & con,ang & 78.21 \\
sur,neu & 92.13 & dis,fea & 88.84 & dis,sad & 84.11 & sad,fea & 76.69 \\
hap,neu & 91.94 & con,fea & 88.13 & sur,sad & 82.86 & ang,dis & 74.20 \\
dis,neu & 91.06 & con,neu & 80.06 & con,dis & 81.61 & fea,sur & 74.11 \\
\hline
\end{tabular}

TABLE III: Performance dynamics of expressive pairs. Each rate is an average of all the experiments using the proposed SVM method for that particular pair of expressions.

\section{G. Favourable results compared to other studies}

To compare with other relevant studies we examined the best results of facial classification studies using the Cohn-Kanade and MMI datasets. For the Cohn-Kanade dataset, the studies that employed six expression classes (the typical 6 - happy, sad, surprised, fearful, angry and disgusted) achieved $93.85 \%$ [48] and 93.8\% [26]; those with seven expression classes (the typical 6 plus neutral) achieved 93.8\% [28] and 93.3\% [4]. For the MMI dataset, the studies that employed six expression classes achieved $82.68 \%$ [44]; those with seven expression 
classes (once again, the typical 6 plus neutral) achieved $86.9 \%$ [40] and $93.61 \%$ [24].

In comparison, our best results employing six expression classes achieved $80.83 \%$ via a component approach, and for seven expression classes we achieved $98.33 \%$ via a component action approach. Extending our previous publication [20], one main difference between our results and the mentioned studies is that we use a 10 fold cross validation algorithm while some use a separate training and test set [26], and others use a different cross validation method ([48] used 2 fold, $[4,24,28,44]$ used leave-one-out). The use of a separate training and test set or a fold lower than 10 will return a higher classification rate.

\section{CONCLUSiON}

In a previous publication we showed a component approach performing better than a holistic approach for classifying 6 discrete expressions of emotion (happy, sad, surprised, fearful, disgusted and angry) plus the neutral face. The significance of that study was to provide a thorough understanding of the choices that impact a classifier's performance and the performance dynamics between expression pairs. As an extension of our previous publication, we justify the use of the contemptuous expression by noting its universal presence in human expression and its asymmetric nature, which is not shared with other expressions.

Our results showed that including the contemptuous expression (as well as the neutral face) leads to a decreased performance in classifying the discrete expressions. Our comparison with the literature shows that the reported classification rates will be artificially higher for studies that exclude these expressions as well as using separate training and testing sets or a cross validation fold lower than 10. Further, our literature review reveals the influence of holistic approaches for facial recognition over component approaches for facial expression classification. And finally, when we analysed the performance of expression pairs, the finding agrees with our previous publication; which is, the performance dynamics of paired expressions suggest a non-equidistant expression space.

\section{REFERENCES}

[1] Studio encoding parameters of digital television for standard $4: 3$ and wide-screen 16:9 aspect ratios, 2007.

[2] R.R. Avent, Chong Teck Ng, and J.A. Neal. Machine vision recognition of facial affect using backpropagation neural networks. In 16th Annual International Conference of the IEEE Engineering in Medicine and Biology Society, 1994. Engineering Advances: New Opportunities for Biomedical Engineers, volume 2, pages 1364-1365, 1994.

[3] W.A. Barrett. A survey of face recognition algorithms and testing results. In Conference Record of the Thirty-First Asilomar Conference on Signals, Systems \& Computers, volume 1, pages 301-305, 1997.

[4] M.S. Bartlett, G. Littlewort, M. Frank, C. Lainscsek, I. Fasel, and J. Movellan. Recognizing facial expression: machine learning and application to spontaneous behavior. In Computer Vision and Pattern Recognition, 2005. CVPR 2005. IEEE Computer Society Conference on, volume 2, pages 568-573, 2005.

[5] M. Beszedes and P. Culverhouse. Comparison of human and automatic facial emotions and emotion intensity levels recognition. 5th International Symposium on Image and Signal Processing and Analysis, pages 429-434, 2007.

[6] Stephan K. Chalup, Kenny Hong, and Michael J. Ostwald. A face-house paradigm for architectural scene analysis. In Richard Chbeir, Youakim
Badr, Ajith Abraham, Dominique Laurent, and Fernnando Ferri, editors, CSTST 2008: Proceedings of The Fifth International Conference on Soft Computing as Transdisciplinary Science and Technology, pages 397403. ACM, 2008

[7] Chih-Chung Chang and Chih-Jen Lin. LIBSVM: a Library for Support Vector Machines, 2001

[8] Chih-wei Hsu and Chih-Jen Lin. A comparison of methods for multiclass support vector machines. IEEE Transactions on Neural Networks, 13(2):415-425, 2002.

[9] Chao-Fa Chuang and Frank Y. Shih. Recognizing facial action units using independent component analysis and support vector machine. Pattern Recognition, 39(9):1795-1798, 2006.

[10] P Ekman and W Friesen. Constants across cultures in the face and emotion. Journal of Personality and Social Psychology, 17(2):124-129, 1971.

[11] P Ekman and W.V Friesen. Facial Action Coding System: A Technique for the Measurement of Facial Movement. Consulting Psychologists Press, 1978.

[12] P. Ekman and K. G. Heider. The universality of a contempt expression: A replication. Motivation and Emotion, 12(3):303-308, 1988.

[13] Paul Ekman. Strong evidence for universals in facial expressions: A reply to russell's mistaken critique. Psychological Bulletin, 115(2):268 287, 1994.

[14] Paul Ekman. Handbook of Cognition and Emotion, chapter 16, pages 301-320. New York: John Wiley and Sons Ltd, 1999.

[15] Paul Ekman. Emotions Revealed. Times Books, Henry Holt and Company 115 West 18th Street New York, New York 10011, 2003.

[16] Paul Ekman and Wallace V. Friesen. A new pan-cultural facial expression of emotion. Motivation and Emotion, 10(2):159-168, 1986.

[17] B. Fasel and Juergen Luettin. Automatic facial expression analysis: A survey. Pattern Recognition, 36(1):259-275, 2003.

[18] Guodong Guo and C.R. Dyer. Learning from examples in the small sample case: face expression recognition. IEEE Transactions on Systems, Man, and Cybernetics, Part B, 35(3):477-488, 2005.

[19] K. Hong, S.K. Chalup, and R.A.R. King. An experimental evaluation of pairwise adaptive support vector machines. In The 2012 International Joint Conference on Neural Networks (IJCNN), pages 1-8, 2012.

[20] Kenny Hong, Stephan Chalup, and Robert King. A component based approach improves classification of discrete facial expressions over a holistic approach. In 2010 International Joint Conference on Neural Networks (IJCNN 2010), pages 90-97. IEEE, 2010.

[21] Alejandro Jaimes and Nicu Sebe. Multimodal human-computer interaction: A survey. Computer Vision and Image Understanding, 108(12):116-134, 2007

[22] S. Kaiser and T. Wehrle. Automated coding of facial behavior in human-computer interactions with facs. Journal of Nonverbal Behavior, 16(2):67-83, 1992.

[23] T. Kanade, J.F. Cohn, and Yingli Tian. Comprehensive database for facial expression analysis. In Proceedings of Fourth IEEE International Conference on Automatic Face and Gesture Recognition, pages 46-53, 2000.

[24] Marios Kyperountas, Anastasios Tefas, and Ioannis Pitas. Salient feature and reliable classifier selection for facial expression classification. Pattern Recognition, 43(3):972-986, 2010.

[25] Andreas Lanitis, Chris J. Taylor, and Timothy F. Cootes. Automatic interpretation and coding of face images using flexible models. IEEE Transactions on Pattern Analysis and Machine Intelligence, 19(7):743756, 1997.

[26] Ying li Tian. Evaluation of face resolution for expression analysis. In Conference on Computer Vision and Pattern Recognition Workshop CVPRW '04, pages 82-82, 2004.

[27] Shu Liao, Wei Fan, A.C.S. Chung, and Dit-Yan Yeung. Facial expression recognition using advanced local binary patterns, tsallis entropies and global appearance features. In IEEE International Conference on Image Processing, pages 665-668, 2006.

[28] Gwen Littlewort, Marian Stewart Bartlett, Ian Fasel, Joshua Susskind, and Javier Movellan. Dynamics of facial expression extracted automatically from video. In Image and Vision Computing, pages 615-625, 2004.

[29] Wei-feng Liu, Ji-li Lu, Zeng-fu Wang, and Hua-jun Song. An expression space model for facial expression analysis. In Congress on Image and Signal Processing, 2008. CISP '08, volume 2, pages 680-684, 2008.

[30] P. Lucey, J.F. Cohn, T. Kanade, J. Saragih, Z. Ambadar, and I. Matthews The extended cohn-kanade dataset (ck+): A complete dataset for action 
unit and emotion-specified expression. In Computer Vision and Pattern Recognition Workshops (CVPRW), 2010 IEEE Computer Society Conference on, pages $94-101,2010$.

[31] Michael J. Lyons, Shigeru Akamatsu, Miyuki Kamachi, and Jiro Gyoba. Coding facial expressions with gabor wavelets. In Proceedings of Third IEEE International Conference on Automatic Face and Gesture Recognition, pages 200-205, 1998.

[32] D. Matsumoto. More evidence for the universality of a contempt expression. Motivation and Emotion, 16(4):363-368, 1992.

[33] Sungsoo Park and Daijin Kim. Subtle facial expression recognition using motion magnification. Pattern Recognition Letters, 30(7):708-716, 2009.

[34] Paul Ekman Group LLC. JACFEE and JACNEUF dataset http://www.humintell.com/for-use-in-research/.

[35] G.I. Roisman, J.L. Tsai, and K.S. Chiang. The emotional integration of childhood experience: Physiological, facial expressive, and selfreported emotional response during the adult attachment interview. Developmental Psychology, 40:776-789, 2004.

[36] J. A. Russell. Is there universal recognition of emotion from facial expression? a review of the cross-cultural studies. Psychological Bulletin, 115:102-141, 1994.

[37] Christopher P. Said, Nicu Sebe, and Alexander Todorov. Structura resemblance to emotional expressions predicts evaluation of emotionally neutral faces. Emotion, 9(2):260-264, 2009.

[38] Ashok Samal and Prasana A. Iyengar. Automatic recognition and analysis of human faces and facial expressions: a survey. Pattern Recognition, 25(1):65-77, 1992.

[39] Fabio Sambataro, Savino Dimalta, Annabella Di Giorgio, Paolo Taurisano, Giuseppe Blasi, Tommaso Scarabino, Giuseppe Giannatempo, Marcello Nardini, and Alessandro Bertolino. Preferential responses in amygdala and insula during presentation of facial contempt and disgust European Journal of Neuroscience, 24:2355-2362, 2006.

[40] Caifeng Shan, Shaogang Gong, and Peter W. McOwan. Facial expression recognition based on local binary patterns: A comprehensive study. Image and Vision Computing, 27(6):803-816, 2009.

[41] V. N. Vapnik. Statistical learning theory. John Wiley \& Sons, New York, 1998.

[42] F. Wallhoff, B. Schuller, M. Hawellek, and G. Rigoll. Efficient recognition of authentic dynamic facial expressions on the feedtum database. 2006 IEEE International Conference on Multimedia and Expo, pages 493-496, 2006.

[43] Hao Wang and Kongqiao Wang. Affective interaction based on personindependent facial expression space. Neurocomputing, 71(10-12):18891901, 2008

[44] Jun Wang and Lijun Yin. Static topographic modeling for facial expression recognition and analysis. Computer Vision and Image Understanding, 108(1-2):19-34, 2007.

[45] Xudong Xie and Kin-Man Lam. Facial expression recognition based on shape and texture. Pattern Recognition, 42(5):1003-1011, 2009.

[46] Zhihong Zeng, Maja Pantic, Glenn I. Roisman, and Thomas S. Huang. A survey of affect recognition methods: Audio, visual, and spontaneous expressions. IEEE Transactions on Pattern Analysis and Machine Intelligence, 31(1):39-58, 2009.

[47] Yongmian Zhang and Qiang Ji. Active and dynamic information fusion for facial expression understanding from image sequences. IEEE Transactions on Pattern Analysis and Machine Intelligence, 27(5):699714,2005

[48] Guoying Zhao and Matti Pietikäinen. Boosted multi-resolution spatiotemporal descriptors for facial expression recognition. Pattern Recognition Letters, 30(12):1117-1127, 2009. 